\title{
Assessment of selected parameters of placental microstructure in patients with intrahepatic cholestasis of pregnancy
}

\author{
Milena Gruszczynska-Losy ${ }^{1}$, Ewa Wender-Ozegowska² ${ }^{2}$ Przemyslaw Wirstlein ${ }^{2}$, \\ Malgorzata Szczepanska² \\ ${ }^{1}$ Gynecologic and Obstetrical University Hospital in Poznan, Poland \\ ${ }^{2}$ Division of Reproduction, Department of Obstetrics, Gynaecology and Gynaecologic Oncology, \\ Poznan University of Medical Sciences, Poznan, Poland
}

\begin{abstract}
Objectives: Intrahepatic cholestasis of pregnancy (ICP) is the most common liver disorder during pregnancy. Cholestasis is associated with increased risk of fetal complications: prematurity, perinatal hypoxia and meconium stained amniotic fluid, and sudden intrauterine fetal death. The exact mechanisms associated with cholestasis fetal sequelae are not fully understood. The aim of the study was the histopathological evaluation of placentas from patients with cholestasis and healthy pregnant women to establish whether cholestasis is accompanied by changes in placental microstructure.

Material and methods: The effect of cholestasis on placental microstructure was investigated using placental tissue from patients with cholestatsis treated with ursodeoxycholic acid (UDCA) and from uncomplicated pregnancies. Five placental histopathological features were analyzed: number of syncytial knots, number of capillaries per villous, structure of stroma, presence of Hofbauer cells, and villitis of unknown etiology.

Results: There were no statistically significant differences in any of the studied parameters between cholestasis-affected and healthy control groups.

Conclusions: There are no diffrences in placental microstructure in cholestasis patients treated with UDCA and in patients with uncomplicated pregnancy.

Key words: intrahepatic cholestasis of pregnancy; ursodeoxycholic acid; placenta; histology
\end{abstract}

Ginekologia Polska 2019; 90, 8: 452-457

\section{INTRODUCTION}

Intrahepatic cholestasis of pregnancy is the most common liver disorder during pregnancy, occurring in $1 \%$ of pregnancies. Very frequently ICP develops during the late second and third trimesters. Pregnant women with cholestasis present characteristic symptoms: pruritus, especially during the night, associated with abnormal liver function. These signs usually resolve after delivery. Among others, the most important biochemical feature of ICP is elevated serum bile acid levels (> $10 \mathrm{mmol} / \mathrm{L}$ ) [1]. Cholestasis is associated with increased risk of fetal complications: prematurity, perinatal hypoxia and meconium stained amniotic fluid, and sudden intrauterine fetal death [2]. The increased risk of detrimental perinatal results correlates with high bile acid concentration (> $40 \mathrm{mmol} / \mathrm{L}$ ) [3]. The exact mechanisms associated with cholestasis fetal sequelaes are not fully understood. In the normal course of pregnancy, the total concentration of bile acids in fetal serum is only sligthly higher than in maternal serum. Because the fetal hepatobiliary and renal systems are not fully developed and cannot eliminate bile acids, the latter must be transferred across the placenta to be eliminated by the maternal liver. In patients with cholestasis, as a result of high levels of bile acids in the serum, the transplacental bile acid gradient is reversed, causing impairment placental transport $[1,4]$.

Bile acids induce vasoconstriction of the chorionic vessels in the placenta that causes impaired fetal-maternal transport across the placenta, damage of the placental structure and reduced transport of nutrients and oxygen to the fetus. 
Little is known about the effect of intrahepatic cholestasis of pregnancy on the placental structure. Most reports involving sudden intrauterine fetal death are not connected with prominent morphological changes in the placenta or the features of its chronic insufficiently $[5,6]$.

The aim of the study was to evaluate the histopathological changes of placentas from patients with cholestasis and healthy pregnant women to establish whether cholestasis affects placental microstructure.

\section{MATERIAL AND METHODS}

Twenty patients with intrahepatic cholestasis and 40 women as controls with physiological pregnancy who delivered in 2018 in the Gynecologic and Obstetrical University Hospital, Poznan, Poland were qualified to this prospective case-control study. Recognision of cholestasis was determined after excluding other liver diseases (viral hepatitis, acute fatty liver of pregnancy, pre-eclampsia, primary biliary cirrhosis and HELLP syndrome), on the basis of unexplained itching associated with increased bile acid (>10 mmol/L) and liver dysfunctions. We also excluded patients with complications of pregnancy other than ICP.We included patients with physiological pregnancy in the control group. Patients in the control group did not have cholestasis in their previous pregnancy or pruritus in the previous or current pregnancy. Inclusion criteria for pregnant women with cholestasis and controls demanded that all patients have single, live-born newborn.

All participants gave written informed consent. The obtained data included concentration of transaminases and bile acid at the time of diagnosis and delivery, gestational week at diagnosis and delivery, the highest concentration of bile acid and transaminases, neonatal birth weight, umbilical pH value, Apgar score, placental weight, and placental/neonatal birth weight ratio (Tab. 1). All women with ICP were treated with ursodeoxycholic acid (UDCA). All the participants were nonsmokers.
The study was approved by the Ethical Committee of Poznan University of Medical Sciences.

\section{Collection of samples and slide preparation}

After delivery placentas were weighed and fixed in $4 \%$ buffered formalin for 10 days. After fixation, the samples were dehydrated using in sequence: $70-100 \%$ series of ethyl alcohol dilutions, xylene, and embedded in $58^{\circ} \mathrm{C}$ paraffin. Samples were cut at $5 \mathrm{~mm}$ using a microtome (Leica SM 2010R) and mounted on slides. The slides were incubated for $2 \mathrm{~h}$ at $58^{\circ} \mathrm{C}$ to remove excess paraffin and ensure laminar adhesion. Deparaffinization was performed in three changes of xylene for 10 min each. Next, the slides were rehydrated through a $100-70 \%$ series of ethyl alcohol dilutions and washed in distilled water. Finally, deparaffinized samples were stained with hematoxylin and eosin (HE) and examined with a light microscope with camera attachment (Axioskop 40 ZEISS) with $100 x$ and $400 x$ magnification.

\section{Histology}

Histological analysis of the preparations ( $n=20$ ICP patients, $n=40$ control patients) was performed by pathologist unaware of gestational age and disease status.

\section{Evaluation of syncytial knots}

The definition of syncytial knots was described by Geenes et al. [7] as the presence of at least 10 aggregated syncytiotrophoblast nuclei that were not in direct contact with communicating villi surfaces. They illustrate areas of intensified apoptosis. In pregnancies with a pathological course complicated by intrauterine growth restriction and pre-eclampsia the amount of syncytial knots enhances. This phenomenon is a result of increased placental apoptosis. Exposure placental explant patches to influence of hyperoxia, hypoxia or reactive oxygen species in vitro causes creation of syncytial knots [7].

Table 1. Characteristics of maternal and delivery outcomes: values expressed as median (range) or mean ( \pm SD)

\begin{tabular}{|l|l|l|l|}
\hline Maternal characteristics & ICP $(\mathbf{n}=\mathbf{2 0})$ & Control $(\mathbf{n}=\mathbf{4 0})$ & P value \\
\hline Age [years] & & & \\
\hline Gravity & $30( \pm 5)$ & $30( \pm 5)$ & 0.869 \\
\hline Parity & $1(1-6)$ & $(1-4)$ & 0.402 \\
\hline Fetal characteristics & $0-5$ & $0-3$ & 0.415 \\
\hline Gestation age at delivery [weeks] & & & 0.698 \\
\hline Birth weight [g] & $37( \pm 2)$ & $38( \pm 2)$ & $\mathbf{0 . 0 4 3}$ \\
\hline Apgar score 5 minutes & $2892( \pm 683)$ & $3623( \pm 2763)$ & 0.058 \\
\hline Umbilical artery pH & $10(5-10)$ & $10(8-10)$ & 0.516 \\
\hline Placental weight [g] & $7.3(7.2-7.5)$ & $7.3(7.1-7.5)$ & 0.164 \\
\hline Placenta/naonatal birth weight ratios & $529( \pm 42)$ & $544( \pm 68)$ & $\mathbf{0 . 0 0 1}$ \\
\hline
\end{tabular}


In each placental sample, in three fields of view, the number of syncytial knots was counted manually. A count of syncytial knot density per $\mathrm{mm}^{2}$ of villous tissue was allowed by the determination the surface area of villous tissue in the frames.

\section{Evaluation of Hofbauer cells (HBCs)}

Hofbauer cells are fetal origin [8]. They are placental villous macrophages, which emerge in placental tissue from 18 days after conception and persist to the end of pregnancy. By the fourth to fifth month of pregnancy, their identification becomes difficult as villous stroma becomes compressed [9]. Functions of tissue macrophages are phagocytosis of cellular debris and antigen presentation in response to infectious agents and inflammation [10]. Due to villitis of unknown etiology (VUE) and varial infection HBCs proliferation or hyperplasia is observed [11, 12]. In placentas from complicated pregnancies (gestational diabetes mellitus, intrauterine growth restriction, pre-eclampsia), the number of HBCs seems to increase while in placentas from uncomplicated pregnancies, HBCs either disappear or become less in number after the fourth month of pregnancy [13].

$\mathrm{HBC}$ s were identified as round or ovoid cells with eccentric nuclei and granular cytoplasm. The HBCs were counted in two high-power fields per slide at $400 \times$ magnification from three different fields in each section. The mean value per villous was calculated (Tab. 2).

\section{Villitis}

Villitis arises due to hematogenous infection of the placenta by TORCH infection. In contrast, majority of cases are immune mediated and are not an effect of infection [14]. There are two types of villitis: acute and chronic.In the first type of villitis, which is often a result of infection, polymorphonuclear leukocytes infiltrate the villi with or without associated necrosis. Whereas in the second type of villitis the tissue is infiltrated by macrophages and lymphocytes usually with concomitant fibrosis and cellular proliferation of the villi $[15,16]$.

\section{Statistical Analysis}

For statistical analysis, SigmaStat version 3.5 software (Systat Software, Inc., Point Richmond, CA, USA) was used. The analysis of the results was based on the Student's t-test for variables with parametric distributions. For variables with non-parametric distributions, the Mann-Whitney rank sum test was used. The Fisher exact test was used for assessment of the villitis distribution. $\mathrm{P}<0.05$ was considered statistically significant.

\section{RESULTS}

Biochemical characteristics of the ICP patient's population is shown in the Table 3. There were no differences in neonatal,maternal and obstetrical outcomes. Nevertheless, there was a statistically significant difference in birth weights ( $2892 \pm 683 \mathrm{~g}$ vs $3623 \pm 2763 \mathrm{~g}, \mathrm{p}=0.043$ ) and placenta/neonatal birth weight ratios ( 0.16 vs $0.18, p=0.001$ ) in neonates born to women with and without cholestasis, respectively (Tab. 1).

The histological slides of 20 patients with ICP and 40 healthy controls were examined. Placentas from both groups were found to be appropriate for their gestational age. Five placental histopathological features were analyzed: number of syncytial knots, number of capillaries per villous, structure of stroma, presence of HBCs, and VUE. The placentas from both groups exhibit normal microstructure: compact stroma and appropriate number of capillaries per villous $(<10)$.

There were no statistically significant differences in any of the remaining three parameters between the cholestasis and healthy control groups (Tab. 3). Representative images are shown in Figure 1.

\begin{tabular}{|l|l|l|l|}
\hline Table 2. Placental histology of the study population & \multicolumn{1}{l|}{} \\
\hline & ICP $(\mathbf{n = 2 0})$ & Control $(\mathbf{n}=\mathbf{4 0})$ & P value \\
\hline Hofbauer cells/villous [median (range)] & $2(0-5)$ & $1(0-3)$ & 0.397 \\
\hline Syncytial knots [median (range)] & $21(12-34)$ & $15(9-29)$ & 0.143 \\
\hline Structure of stroma & compact & compact & - \\
\hline Villitis of unknown etiology & $1(5 \%)$ & $3(7.5 \%)$ & 0.999 \\
\hline Number of capillaries per villous & $<10$ & $<10$ & - \\
\hline
\end{tabular}

\begin{tabular}{|c|c|c|c|c|c|}
\hline Sample & Week of delivery [week] & $\begin{array}{l}\text { Bile acid } \\
\text { (highest value) [mmol/L] }\end{array}$ & $\begin{array}{l}\text { Alat } \\
{[U / L]}\end{array}$ & $\begin{array}{l}\text { Aspat } \\
{[U / L]}\end{array}$ & $\begin{array}{l}\text { Bile acid at delivery } \\
\text { [mmol/L] }\end{array}$ \\
\hline$N=40$ & $37( \pm 2)$ & $\begin{array}{l}37.5 \\
(11-171.3)\end{array}$ & $\begin{array}{l}188.1 \\
(13.5-1228.9)\end{array}$ & $\begin{array}{l}101.2 \\
(16.7-695.2)\end{array}$ & $\begin{array}{l}22.4 \\
(10.8-102)\end{array}$ \\
\hline
\end{tabular}


A

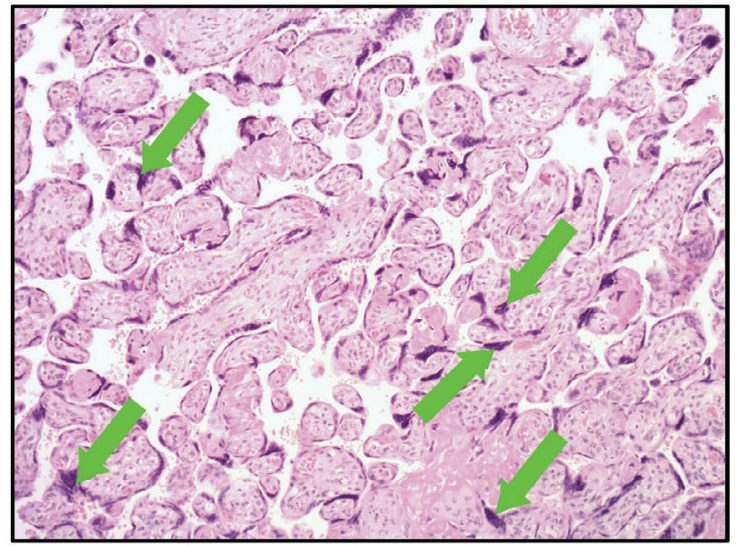

A1

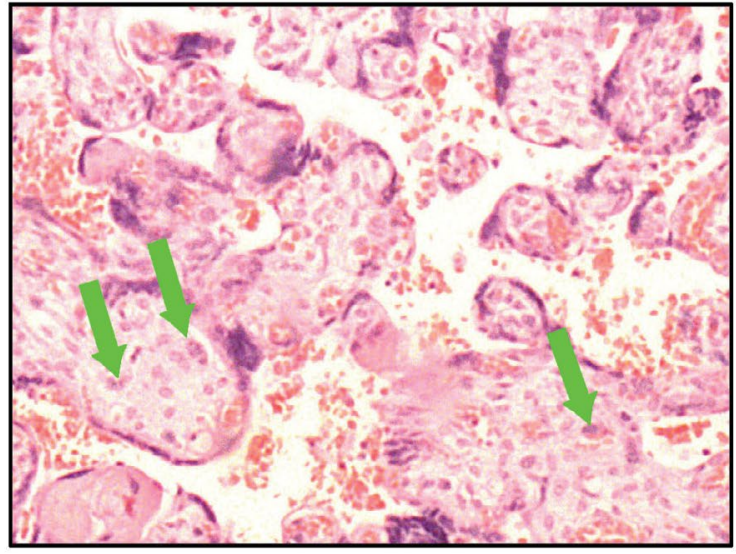

B

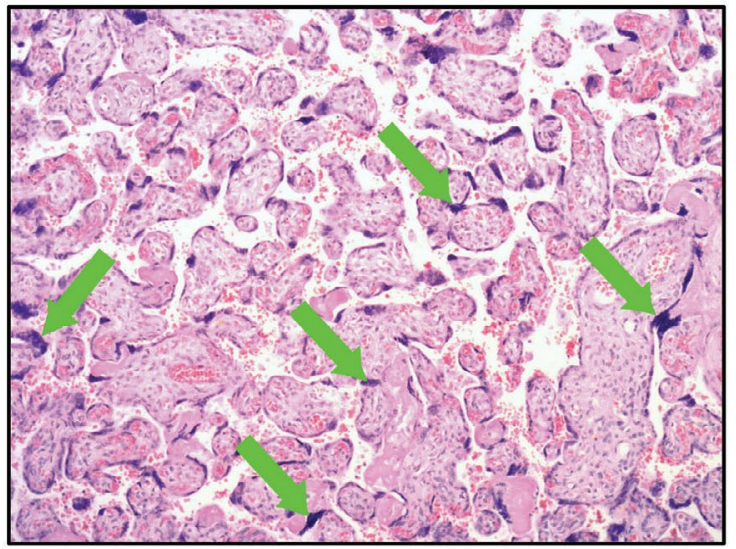

B1

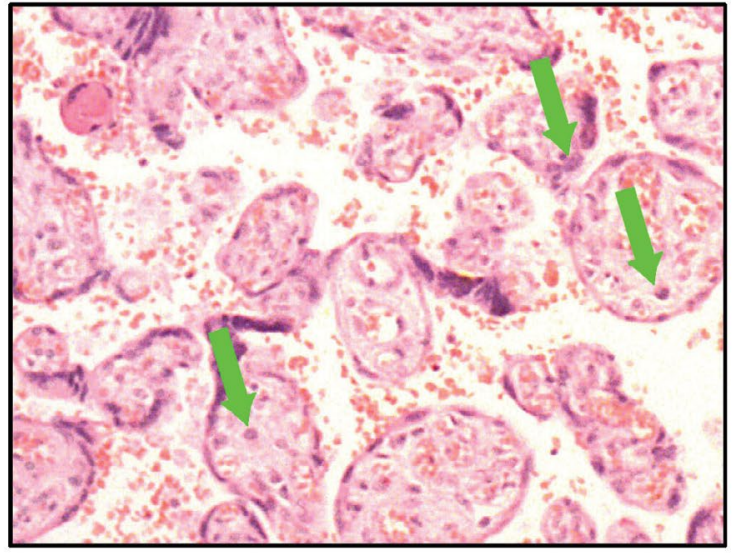

Figure 1. Representative images villi of trophoblast in a $20 \times(\mathbf{A}, \mathbf{B})$ and $40 \times(\mathbf{A 1}, \mathbf{B} 1)$ lens magnification microscope stained by $H+E$ obtained from women with normal pregnancy $(\mathbf{A}, \mathbf{A} 1)$ and with pregnancy complicated by cholestasis (B, B1) treated with ursodeoxycholic acid. The green arrows indicate the syncytial knots (A, B) and Hofbauer cells (A1, B1).

\section{DISCUSSION}

In this paper, we analyzed the histopathological images of placentas of patients with pregnancy complicated with cholestasis, and non-complicated, phisiological pregnancy. All pregnant women with cholestasis were treated with UDCA which is considered the first-line treatment for ICP, because it normalizes the transplacental bile acid gradient, significantly reducing fetal and maternal bile acid levels [17]. Five placental histopathological features were analyzed: number of syncytial knots, number of capillaries per villous, structure of stroma, presence of HBCs and VUE. In our study, we did not find any differences in the microstructure of the placenta of patients with cholestasis relative to patients with normal pregnancy.

Our results are in accordance with Patel et al. [18] who compared the placentas of 24 pregnant women with ICP and 30 healthy women. They found no differences in maternal, neonatal, and obstetrical outcomes. They found a significant difference between the groups only in the gestation age at delivery. Patients with cholestasis delivered two weeks earlier than the controls. The authors analyzed 17 placental histopathological parameters and didn't find statistically significant differences in any of these features between patients with cholestasis and healthy pregnant women. Comparison of the placentas from women, treated and not treated with UDCA, demonstrated a statistically significant reduction in VUE ( $9 \%$ vs $53 \%, p=0.03$ ), which implies that UDCA has an anti-inflammatory impact on the placenta. However, the exact mechanism by which UDCA decreases placental inflammation is not known.

Guven et al. [19] investigated the alterations in the architecture of the umbilical cord and the placenta in selected pregnancy complications. They stated a significant enhancement in the amount of syncytial knots in placentas from women with pre-eclampsia, oligohydramnions, polyhydramnions, and repeated cesarean sections, but not in ICP 
and control groups. Furthermore, they found a significant reduction in the diameter and volume of the arterial lumen of an umbilical artery in the ICP group compared to the control group. The arterial tunica intima and tunica media were thicker in ICP than in the control group.

The available data of the histopathological analysis of placenta in patients with cholestasis and its comparison with that of the group of healthy pregnant women indicates morphological differences. These changes include higher amount of syncytial knots and enhancement surface of terminal villi, and were observed in pregnant women with cholestasis untreated with UDCA.

In the article of Geenes et al. [20] slides prepared from 28 ICP patients and 12 healthy controls were subjected to histological examination. In the placentas achieved from pregnant women with cholestasis a couple of morphological abnormalities were found to be more frequent as compared to the placentas from physiological pregnancies. These contained chorionic villi that had dense fibrotic stroma and were small for the gestational age, focally-thickened amniotic basement membranes, increased in number of syncytial knots and stricture of intervillous space. The number of syncytial knots was significantly higher in placentas from untreated women with ICP compared to those from uncomplicated pregnancies ( $p=0.02$ ). However, there was no significant difference in the amount of syncytial knots in the placentas achieved from women with cholestasis treated with UDCA compared to healthy pregnant women.

Wikström Shemer et al. [21] in a prospective case-control study using the computerized stereology method, examined placentas from 10 untreated and 10 UDCA-treated patients with cholestasis, and eight healthy pregnant women for morphological differences. They analyzed five histopathological features of placentas (volume fraction of collagen, surface area of terminal villi and capillaries, chorangiosis,anumber of syncytial knots and volume of placenta).

The results of their research demonstrated that cholestasis influences the microarchitecture of placenta by enhancement the number of syncytial knots and terminal villous and capillary surface area. The UDCA treatment had protective effect on placental micro architecture. The analyses of placentas of UDCA-treated cholestasis, compared to untreated ICP, showed statistically significant differences in amount of syncytial knots, capillary surface area and terminal villous. The statistical differences were not detected in any analyzed parameters in placentas between UDCA-treated cholestasis patients and healthy pregnant women.

Increased capillary growth in terminal villi and syncytial knots were both described as a sign of hypoxia which is commonly evident in placentas coexisting with diseases with reduced blood perfusion (diabetes mellitus and pre-eclampsia).
Conclusions from the two abovementioned cited studies indicate that ICP is associated with a couple of anomalies of the placenta microstructure, including an enhancement in the amount of syncytial knots. However, in placentas of women treated with UDCA, the number of syncytial knots was comparable to those in placentas from uncomplicated pregnancies $[20,21]$.

The studies of Geenes et al. [20], Wikström Shemer et al. [21], and Patel et al. [18] were published in 2011, 2012, and 2014 respectively and, probably for that reason, some patients did not receive treatment with UDCA which is currently the first-line treatment for cholestasis.

The final answer to the question, whether treatment with UDCA in women with cholestasis improves obstetric outcomes, will be obtained after the completion of the triple-masked, placebo-controlled, randomised trial - PITCHES (Phase III trial in IntrahepaTic CHolestasis of pregnancy to Evaluate urSodeoxycholic acid in improving perinatal outcomes) which is currently randomized [22].

Our study has a number of limitations. Our work was carried out on a relatively small group of patients, but the real cholestasis, confirmed with biochemical data is rather rare in our population. To reduce statistical error, the histological examination of placentas should be carried out on a larger and an independent cohort. All patients with cholestasis included in this study, as well as all patients with cholestasis who delivered in 2018 in Gynecologic and Obstetrical University Hospital in Poznan, were treated with UDCA before delivery. UDCA treatment of pregnant women with cholestasis is standard in our hospital; therefore it was not possible to compare placental microstructure between patients with pregnancy complicated by cholestasis treated and not treated with UDCA.

\section{CONCLUSIONS}

Based on obtained results, we can conclude that the histopathological structure of the placenta of patients with cholestasis treated with UDCA does not differ from women with normal pregnancy.

\section{REFERENCES}

1. Mcllvride S, Dixon PH, Williamson C. Bile acids and gestation. Mol Aspects Med. 2017; 56: 90-100, doi: 10.1016/j.mam.2017.05.003, indexed in Pubmed: 28506676.

2. Geenes V, Chappell LC, Seed PT, et al. Association of severe intrahepatic cholestasis of pregnancy with adverse pregnancy outcomes: a prospective population-based case-control study. Hepatology. 2014; 59(4): 1482-1491, doi: 10.1002/hep.26617, indexed in Pubmed: 23857305.

3. Kawakita T, Parikh LI, Ramsey PS, et al. Predictors of adverse neonatal outcomes in intrahepatic cholestasis of pregnancy. Am J Obstet Gynecol. 2015; 213(4): 570.e1-570.e8, doi: 10.1016/j.ajog.2015.06.021, indexed in Pubmed: 26071912.

4. Geenes V, Lövgren-Sandblom A, Benthin L, et al. The reversed feto-maternal bile acid gradient in intrahepatic cholestasis of pregnancy is corrected by ursodeoxycholic acid. PLoS One. 2014; 9(1): e83828, doi: 10.1371/journal.pone.0083828, indexed in Pubmed: 24421907. 
5. Lee $\mathrm{RH}$, Incerpi $\mathrm{MH}$, Miller DA, et al. Sudden fetal death in intrahepatic cholestasis of pregnancy. Obstet Gynecol. 2009; 113(2 Pt 2): 528-531, doi: 10.1097/AOG.0b013e31818db1c9, indexed in Pubmed: 19155945.

6. Ptacek I, Sebire NJ, Man JA, et al. Systematic review of placental pathology reported in association with stillbirth. Placenta. 2014; 35(8): 552-562, doi: 10.1016/j.placenta.2014.05.011, indexed in Pubmed: 24953162

7. Geenes VL, Lim YH, Bowman N, et al. A placental phenotype for intrahepatic cholestasis of pregnancy. Placenta. 2011; 32(12): 1026-1032, doi: 10.1016/j.placenta.2011.09.006, indexed in Pubmed: 22015023.

8. Reyes L, Wolfe B, Golos T. Hofbauer Cells: Placental Macrophages of Fetal Origin. Results Probl Cell Differ. 2017;62:45-60, doi: 10.1007/978-3-31954090-0_3, indexed in Pubmed: 28455705.

9. Ingman K, Cookson VJ, Jones CJP, et al. Characterisation of Hofbauer cells in first and second trimester placenta: incidence, phenotype, survival in vitro and motility. Placenta. 2010; 31(6): 535-544, doi: 10.1016/j. placenta.2010.03.003, indexed in Pubmed: 20347485.

10. Reyes L, Golos TG. Hofbauer Cells: Their Role in Healthy and Complicated Pregnancy. Front Immunol. 2018; 9: 2628, doi: 10.3389/fimmu.2018.02628, indexed in Pubmed: 30498493.

11. Kim JS, Romero R, Kim MR, et al. Involvement of Hofbauer cells and maternal T cells in villitis of unknown aetiology. Histopathology. 2008; 52(4): 457-464, doi: 10.1111/j.1365-2559.2008.02964.x, indexed in Pubmed: 18315598.

12. Kim MiJ, Romero R, Kim CJ, et al. Villitis of unknown etiology is associated with a distinct pattern of chemokine up-regulation in the feto-maternal and placental compartments: implications for conjoint maternal allograft rejection and maternal anti-fetal graft-versus-host disease. J Immunol. 2009; 182(6): 3919-3927, doi: 10.4049/jimmunol.0803834, indexed in Pubmed: 19265171.

13. Saeed I, Yousaf A, Ali S. Number of Hofbauer Cells in Placentae from Normal and Pre Eclamptic Gestation. Journal of Rawalpindi Medical College. 2018; 22(1): 76-78.
14. Bittencourt AL, Garcia AG. The placenta in hematogenous infections. Pediatr Pathol Mol Med. 2002; 21(4): 401-432, doi: 10.1080/152279502760157768, indexed in Pubmed: 12092706.

15. Faye-Petersen OM. The placenta in preterm birth. J Clin Pathol. 2008;61(12): 1261-1275, doi: 10.1136/jcp.2008.055244, indexed in Pubmed: 19074631.

16. Tamblyn JA, Lissauer DM, Powell $R$, et al. The immunological basis of villitis of unknown etiology - review. Placenta. 2013; 34(10): 846-855, doi: 10.1016/j.placenta.2013.07.002, indexed in Pubmed: 23891153.

17. Kong $X$, Kong $Y$, Zhang $F$, et al. Evaluating the effectiveness and safety of ursodeoxycholic acid in treatment of intrahepatic cholestasis of pregnancy: A meta-analysis (a prisma-compliant study). Medicine (Baltimore). 2016; 95(40): e4949, doi: 10.1097/MD.0000000000004949, indexed in Pubmed: 27749550.

18. Patel S, Pinheiro M, Felix JC, et al. A case-control review of placentas from patients with intrahepatic cholestasis of pregnancy. Fetal Pediatr Pathol. 2014; 33(4): 210-215, doi: 10.3109/15513815.2014.899413, indexed in Pubmed: 24758367.

19. Güven D, Altunkaynak BZ, Altun G, et al. Histomorphometric changes in the placenta and umbilical cord during complications of pregnancy. Biotech Histochem. 2018; 93(3): 198-210, doi: 10.1080/10520295.2017.1410993, indexed in Pubmed: 29366351.

20. Geenes V, Lövgren-Sandblom A, Benthin L, et al. The reversed feto-maternal bile acid gradient in intrahepatic cholestasis of pregnancy is corrected by ursodeoxycholic acid. PLoS One. 2014; 9(1): e83828, doi: 10.1371/journal.pone.0083828, indexed in Pubmed: 24421907.

21. Wikström Shemer E, Thorsell M, Östlund E, et al. Stereological assessment of placental morphology in intrahepatic cholestasis of pregnancy. Placenta. 2012; 33(11): 914-918, doi: 10.1016/j.placenta.2012.08.005, indexed in Pubmed: 23020907.

22. Chappell LC, Chambers J, Dixon PH, et al. Ursodeoxycholic acid versus placebo in the treatment of women with intrahepatic cholestasis of pregnancy (ICP) to improve perinatal outcomes: protocol for a randomised controlled trial (PITCHES). Trials. 2018; 19(1): 657, doi: 10.1186/s13063018-3018-4, indexed in Pubmed: 30482254. 